\title{
Comparison of cardiovascular magnetic resonance characteristics and clinical consequences in children and adolescents with isolated left ventricular non-compaction with and without late gadolinium enhancement
}

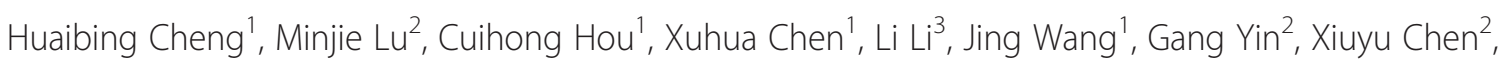
Wei Xiangli ${ }^{2}$, Chen Cui ${ }^{2}$, Jianmin Chu', Shu Zhang ${ }^{1}$, Sanjay K Prasad ${ }^{4}$, Jielin $\mathrm{Pu}^{1 *}$ and Shihua Zhao ${ }^{2^{*}}$

\begin{abstract}
Background: Although cardiovascular magnetic resonance (CMR) is showing increasingly diagnostic potential in left ventricular non-compaction (LVNC), relatively little research relevant to CMR is conducted in children with LVNC. This study was performed to characterize and compare CMR features and clinical outcomes in children with LVNC with and without late gadolinium enhancement (LGE).

Methods: A cohort of 40 consecutive children (age, $13.7 \pm 3.3$ years; 29 boys and 11 girls) with isolated LVNC underwent a baseline CMR scan with subsequent clinical follow-up. Short-axis cine images were used to calculate left ventricular (LV) ejection fraction (EF), end-diastolic volume (EDV), end-systolic volume (ESV), myocardial mass, ratio of non-compacted-tocompacted myocardial thickness (NC/C ratio), and number of non-compacted segments. The LGE images were analyzed to assess visually presence and patterns of LGE. The primary end point was a composite of cardiac death and heart transplantation.

Results: The LGE was present in 10 (25\%) children, and 46 (27\%) segments were involved, including 23 non-compacted segments and 23 normal segments. Compared with LGE-cohort, LGE+ cohort had significantly lower LVEF $(23.8 \pm 10.7 \%$ vs. $42.9 \pm 16.7 \%, p<0.001)$ and greater LVEDV $\left(169.2 \pm 65.1\right.$ vs. $\left.118.2 \pm 48.9 \mathrm{~mL} / \mathrm{m}^{2}, p=0.010\right)$, LVESV $\left(131.3 \pm 55.5\right.$ vs. $\left.73.3 \pm 46.7 \mathrm{~mL} / \mathrm{m}^{2}, p=0.002\right)$, and sphericity indices $(0.75 \pm 0.19$ vs. $0.60 \pm 0.20, p=0.045)$. There were no differences in terms of number and distribution of non-compacted segments, NC/C ratio, and myocardial mass index between LGE+ and LGE- cohort. In the LGE+ cohort, adverse events occurred in 6 patients compared to 2 events in the LGE- cohort. Kaplan-Meier analysis showed a significant difference in outcome between LGE+ and LGE- cohort for cardiac death and heart transplantation $(p=0.011)$.

(Continued on next page)
\end{abstract}

\footnotetext{
*Correspondence: fuwaipujielin@163.com; cjrzhaoshihua@163.com

${ }^{1}$ Center for Arrhythmia Diagnosis and Treatment, State Key Laboratory of Cardiovascular Disease, Fuwai Hospital, National Center for Cardiovascular Diseases, Chinese Academy of Medical Sciences and Peking Union Medical College, 100037 Beijing, People's Republic of China

${ }^{2}$ Department of Radiology, State Key Laboratory of Cardiovascular Disease,

Fuwai Hospital, National Center for Cardiovascular Diseases, Chinese

Academy of Medical Sciences and Peking Union Medical College, 100037

Beijing, People's Republic of China

Full list of author information is available at the end of the article
} 
(Continued from previous page)

Conclusions: The LGE was present in up to one-fourth of children with LVNC, and the LGE+ children exhibited a more maladaptive LV remodeling and a higher incidence of cardiovascular death and heart transplantation.

Keywords: Left ventricular non-compaction, Cardiovascular magnetic resonance, Late gadolinium enhancement, Children

\section{Background}

Left ventricular non-compaction (LVNC) is a genetically and clinically heterogeneous cardiomyopathy characterized by numerous prominent trabeculations, progressive myocardial dysfunction, malignant ventricular arrhythmias, and early mortality $[1,2]$. Left ventricular non-compaction can present in isolation or in combination with other congenital heart diseases or genetic neuromuscular conditions [3-5]. Previous work has shown that LVNC accounts for about $9 \%$ of cardiomyopathy in childhood [6]. The incidence of isolated LVNC diagnosed at children echocardiograms was estimated to be approximately $0.2 \%$ [7].

Cardiovascular magnetic resonance (CMR) may outperform echocardiography in assessing the whole left ventricular (LV) myocardium and also provide precise identification of prominent trabeculations owing to its higher spatial resolution and field of view [8-10]. Furthermore, CMR with late gadolinium enhancement (LGE) is a reliable technique for detecting myocardial fibrosis in vivo, which is related to clinical severity grading and prognosis in adult patients with LVNC [11-15]. Although CMR is showing increasingly diagnostic potential in LVNC, works on the application of CMR in children with LVNC are restricted to several case reports and small case series [16-19]. Therefore, we have assembled a relatively large cohort of children with LVNC, defined by eligibility for CMR, to characterize and compare CMR features and clinical outcomes in children with LVNC with and without LGE.

\section{Methods}

\section{Study patients}

We prospectively recruited a cohort of consecutive children with isolated LVNC who were referred to the Fuwai Hospital for CMR between June 2006 and December 2013. The diagnosis of LVNC was made on the basis of previously defined CMR and clinical criteria: [9] (a) appearance of 2 distinct myocardial layers; (b) prominent myocardial trabeculations and deep intertrabecular recesses communicating with the LV cavity; (c) end-diastolic ratio of non-compacted-to-compacted (NC:C) myocardium $>2.3: 1$, and (d) absence of other known co-existing cardiac abnormalities. The study complied with the Declaration of Helsinki and was approved by the Fuwai Hospital ethics committee, and informed consent was obtained from the parents of each child with LVNC.

\section{CMR image acquisition and analysis}

All CMR exams were performed using a 1.5-T scanner (Siemens Avanto). Retrospective electrocardiographic gated cine images were performed using true fast imaging with steady-state free precession sequence (image parameters: repetition time/echo time $[\mathrm{TR} / \mathrm{TE}]=40.0 / 1.1 \mathrm{~ms}$; matrix $=$ $256 \times 192 \mathrm{~mm}$; flip angle $=62^{\circ}$ ) in three long-axis (horizontal and vertical long-axis and LV outflow tract) and continuous short-axis views covering the entire LV from base to apex. Fifteen $\pm 5 \mathrm{~min}$ after the injection of $0.2 \mathrm{mmol} / \mathrm{kg}$ of gadolinium-DTPA (Magnevist; Schering, Berlin, Germany), the LGE images were obtained using an inversion recovery sequence (image parameters: $\mathrm{TR} / \mathrm{TE}=8.7 / 3.4 \mathrm{~ms}$, matrix $=$ $256 \times 256 \mathrm{~mm}$, flip angle $=15^{\circ}$ ) in three long-axis and standard short-axis views covering the whole LV.

All images were analyzed using a workstation with commercially available software (Siemens Argus). For children with multiple CMR studies, the initial baseline study was used for the primary analysis. Results for LV ejection fraction, ventricular volumes, and myocardial mass were derived from short-axis slices. As previously demonstrated, the papillary muscles were excluded from compacted myocardium because it is difficult to differentiate papillary muscles from dense trabeculations [20]. Left ventricular volumes and compacted mass were indexed to body surface area. The LV sphericity index was calculated as: end-diastolic volume (EDV)/([end-diastolic longaxis diameter $\left.{ }^{3} \times \pi\right] / 6$ ) [21]. The presence or absence of non-compaction and LGE was qualitatively assessed using the AHA 17 segment model [22]. For each segment with non-compaction, the end-diastole $\mathrm{NC} / \mathrm{C}$ ratio was quantitatively calculated in the short-axis views, and the maximum ratio was then used for analysis. As previously demonstrated, the assessment of $\mathrm{NC} / \mathrm{C}$ ratio of the apex (segment 17) was excluded [9]. LGE was deemed present only if myocardial enhancement was confirmed on both short-axis and matching long-axis areas using a signal intensity threshold of $\geq 6$ standard deviations (SD) above a remote reference region (Fig. 1). The myocardial layer distributions of LGE were assessed using the following scale: 1 = subepicardial, 2 = mid-wall, 3 = subendocardial, and $4=$ transmural ( $\geq 75 \%$ LV wall thickness). The LGE score was finally summed. The evaluations of non-compaction and LGE were performed by 2 independent expert readers (ML and $\mathrm{HC}$ ) who were blinded to the clinical data. Discordant findings were resolved by consensus. 


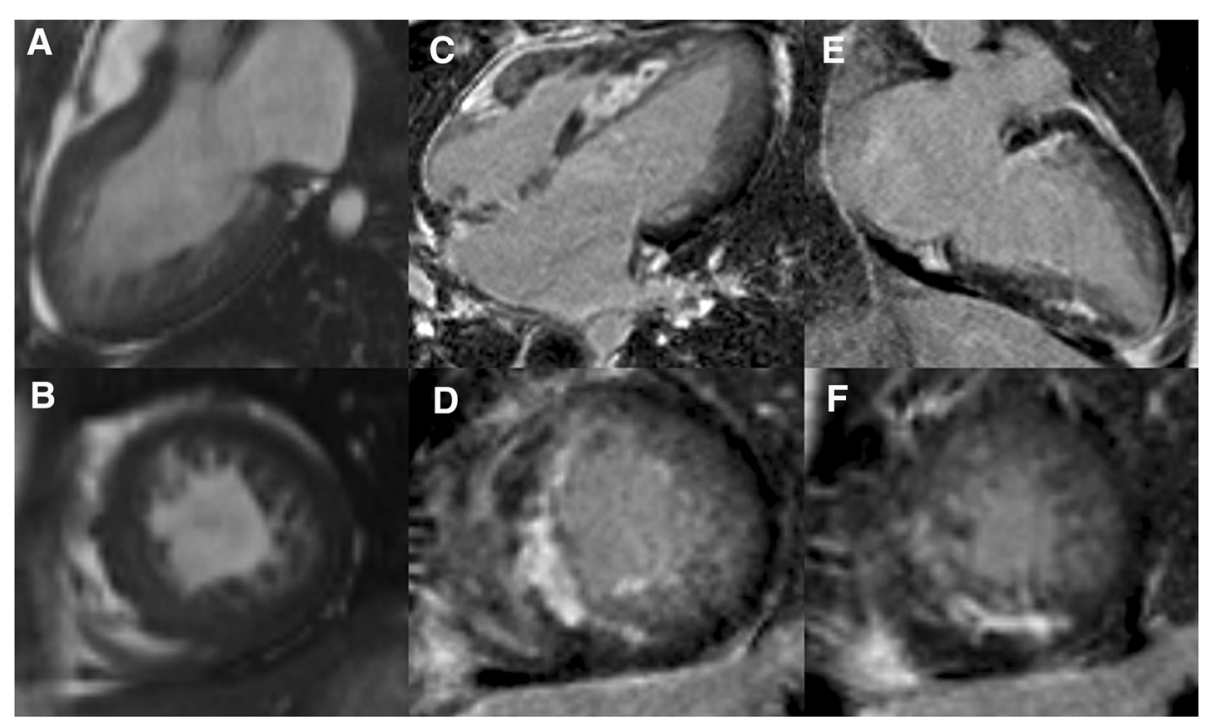

Fig. 1 Representative images of LGE in a 16-year-old boy with LVNC. The vertical (a) and horizontal (c) long-axis and short-axis (b and d) contrast-enhanced images demonstrate transmural LGE in the septal and inferior segments

\section{Follow-up}

All children were followed up via clinic visits or telephone interview after initial CMR examination. The endpoint of the study was cardiovascular death and heart transplantation. The duration of follow-up was determined from the first CMR evaluation date to the occurrence of an endpoint. Complete follow-up was available for all children.

\section{Statistical analysis}

Normality was tested using the Kolmogorov-Smirnov test. Continuous variables were expressed as mean \pm SD and assessed using unpaired t tests or Wilcoxon's rank-sum tests. Categorical variables were expressed as frequencies (percentages) and assessed using the $\chi^{2}$ or Fisher exact tests. Correlation analyses were performed using the Spearman's rank correlation test. Survival curve was generated by the Kaplan-Meier method and compared by the log rank test. The statistical analysis was performed using SPSS for Windows 16.0 (Chicago, IL, USA). A two tailed $\mathrm{p}$ value of $<0.05$ was considered statistically significant.

\section{Results}

\section{Baseline characteristics}

A cohort of 40 children was diagnosed with isolated LVNC. An additional 8 children with prominent trabeculations who met CMR diagnostic criteria were associated with either congenital heart diseases and thus excluded from the study, such as atrial septal defect $(\mathrm{n}=4)$, Ebstein's anomaly $(\mathrm{n}=1)$, aorto-left ventricular tunnel $(\mathrm{n}=1)$, membranous subaortic stenosis $(n=1)$, bicuspid aortic valve $(n=1)$. Twenty-nine children (72\%) were male and 11 (28\%) were female. The mean age at the time of initial CMR scan was $13.7 \pm 3.3$ years (range, $1.5-17$ years). Three children (8\%) had a family history of LVNC. Twenty-one children (53\%) presented primarily with signs and symptoms of congestive heart failure. Seven children (18 \%) presented with documented ventricular arrhythmias and 1 patient (3\%) presented with aborted sudden death. Five children (13\%) were referred for evaluation of unexplained syncope. Two children (5\%) presented with a primary complaint of chest pain. No children had signs of systemic emboli. Clinical and demographic characteristics of the study population are summarized in Table 1.

\section{CMR findings}

The detailed CMR characteristics of children with LVNC are listed in Table 2. The mean LV ejection fraction (EF) and EDV index were $38.1 \pm 17.4 \%$ and $131.0 \pm 55.8 \mathrm{~mL} / \mathrm{m}^{2}$, respectively. No thrombus was detected in LV cavity on CMR. Non-compaction was present in 372 LV segments. The mean number of non-compacted segments per patient was $9.3 \pm 2.5$. The mean $\mathrm{NC} / \mathrm{C}$ ratio was $3.64 \pm 0.94$.

A total of 10 (25\%) children with LVNC showed LV LGE. The LGE was observed in 46 segments, including 23 non-compacted segments and 23 normal segments. Of these, LGE was subendocardial in 11 segments, mid-wall in 18 , subepicardial in 5 , and transmural in 12 . No association was found between myocardial layer distributions of LGE and LVEF $(r=0.49, p=0.148)$. Compared LGE- children, LGE + children had significantly lower LVEF $(23.8 \pm 10.7 \%$ vs. $42.9 \pm 16.7 \%, \mathrm{p}<0.001)$ and greater LVEDV (169.2 \pm 65.1 vs. $\left.118.2 \pm 48.9 \mathrm{~mL} / \mathrm{m}^{2}, \mathrm{p}=0.010\right), \mathrm{LV}$ end-systolic volume $\left(131.3 \pm 55.5\right.$ vs. $73.3 \pm 46.7 \mathrm{~mL} / \mathrm{m}^{2}$, $\mathrm{p}=0.002)$, and sphericity indices $(0.75 \pm 0.19$ vs. $0.60 \pm$ $0.20, \mathrm{p}=0.045)$. There were no differences in terms of number of non-compacted segments, $\mathrm{NC} / \mathrm{C}$ ratio, and 
Table 1 Baseline characteristics

\begin{tabular}{|c|c|c|c|c|}
\hline \multirow[b]{2}{*}{ Variable } & \multirow{2}{*}{$\begin{array}{l}\text { All Children } \\
(n=40)\end{array}$} & \multicolumn{3}{|c|}{ Presence of LGE } \\
\hline & & Yes $(n=10)$ & No $(n=30)$ & p Value \\
\hline Age (years) & $13.7 \pm 3.3$ & $14.0 \pm 2.1$ & $13.6 \pm 3.6$ & 0.753 \\
\hline Male, n (\%) & $29(73)$ & $9(90)$ & $20(67)$ & 0.233 \\
\hline Family history of LVNC, n (\%) & $3(8)$ & $1(10)$ & $2(7)$ & 1.000 \\
\hline \multicolumn{5}{|l|}{ Symptoms } \\
\hline Dyspnoea, n (\%) & $21(53)$ & $9(90)$ & $12(40)$ & 0.004 \\
\hline Chest pain, n (\%) & $2(5)$ & 0 & $2(7)$ & 1.000 \\
\hline Syncope/pre-syncope, n (\%) & $7(18)$ & $1(10)$ & $6(20)$ & 0.651 \\
\hline Thrombo-embolic events, n (\%) & 0 & 0 & 0 & \\
\hline NYHA functional class & $2.4 \pm 1.0$ & $3.0 \pm 0.9$ & $2.1 \pm 0.9$ & 0.016 \\
\hline I, n (\%) & $10(25)$ & $1(9)$ & $9(30)$ & \\
\hline$\|, \mathrm{n}(\%)$ & $11(28)$ & $1(9)$ & $10(33)$ & \\
\hline III, n (\%) & $14(35)$ & $5(55)$ & $9(30)$ & \\
\hline IV, n (\%) & $5(12)$ & $3(27)$ & $2(7)$ & \\
\hline Abnormal ECG, n (\%) & $36(90)$ & $10(100)$ & $26(87)$ & 0.559 \\
\hline VTNF, n (\%) & $7(18)$ & $3(30)$ & $4(13)$ & 0.361 \\
\hline \multicolumn{5}{|l|}{ Medications } \\
\hline$\beta$-Blockers, n (\%) & $29(73)$ & $8(80)$ & $21(70)$ & 0.694 \\
\hline ACEI/ARB, n (\%) & $29(73)$ & $10(100)$ & $19(63)$ & 0.020 \\
\hline Aldosterone antagonists, n (\%) & $27(68)$ & $10(100)$ & $17(57)$ & 0.008 \\
\hline Loop diuretics, n (\%) & $22(55)$ & $9(90)$ & $13(43)$ & 0.011 \\
\hline Amiodarone, n (\%) & $2(5)$ & $2(20)$ & 0 & 0.067 \\
\hline
\end{tabular}

Values are mean \pm SD or $n(\%)$

myocardial mass index between LGE+ and LGE- cohort. There was a similar distribution of non-compaction between LGE+ and LGE- children, which was more frequently observed on the apical segment than on the midcavity and basal segments (Fig. 2).
Follow-up

The average length of follow-up was $3.0 \pm 2.2$ years. During follow-up period, 6 children (15\%) died and 2 (5\%) underwent heart transplantation (Fig. 3), thereby resulting in a $20 \%$ incidence of death and transplantation in the

Table 2 Cardiovascular magnetic resonance characteristics

\begin{tabular}{|c|c|c|c|c|}
\hline \multirow[b]{2}{*}{ Variable } & \multirow{2}{*}{$\begin{array}{l}\text { All Children } \\
(n=40)\end{array}$} & \multicolumn{3}{|c|}{ Presence of LGE } \\
\hline & & Yes $(n=10)$ & No $(n=30)$ & p Value \\
\hline Heart rate (beats/min) & $86.5 \pm 22.8$ & $92.3 \pm 24.8$ & $84.6 \pm 22.2$ & 0.360 \\
\hline Body surface area $\left(\mathrm{m}^{2}\right)$ & $1.6 \pm 0.3$ & $1.5 \pm 0.2$ & $1.6 \pm 0.4$ & 0.861 \\
\hline LV long-axis diameter (mm) & $84.1 \pm 12.0$ & $86.1 \pm 9.3$ & $83.4 \pm 12.8$ & 0.534 \\
\hline LV Sphericity index & $0.64 \pm 0.21$ & $0.75 \pm 0.19$ & $0.60 \pm 0.20$ & 0.045 \\
\hline LVEF (\%) & $38.1 \pm 17.4$ & $23.8 \pm 10.7$ & $42.9 \pm 16.7$ & $<0.001$ \\
\hline LVEDV index $\left(\mathrm{mL} / \mathrm{m}^{2}\right)$ & $131.0 \pm 55.8$ & $169.2 \pm 65.1$ & $118.2 \pm 48.9$ & 0.010 \\
\hline LVESV index $\left(\mathrm{mL} / \mathrm{m}^{2}\right)$ & $87.8 \pm 54.6$ & $131.3 \pm 55.5$ & $73.3 \pm 46.7$ & 0.002 \\
\hline Stroke volume index $\left(\mathrm{mL} / \mathrm{m}^{2}\right)$ & $43.2 \pm 13.4$ & $37.9 \pm 17.0$ & $44.9 \pm 11.8$ & 0.152 \\
\hline Myocardial mass index $\left(\mathrm{mL} / \mathrm{m}^{2}\right)$ & $56.1 \pm 19.0$ & $64.6 \pm 17.2$ & $53.3 \pm 19.0$ & 0.107 \\
\hline Maximum NC/C ratio & $3.64 \pm 0.94$ & $3.76 \pm 1.24$ & $3.60 \pm 0.84$ & 0.645 \\
\hline Number of non-compacted segments & $9.3 \pm 2.5$ & $9.9 \pm 2.8$ & $9.1 \pm 2.4$ & 0.384 \\
\hline Duration of follow-up, years & $3.0 \pm 2.2$ & $2.6 \pm 2.3$ & $3.2 \pm 2.3$ & 0.494 \\
\hline
\end{tabular}

Values are mean \pm SD 


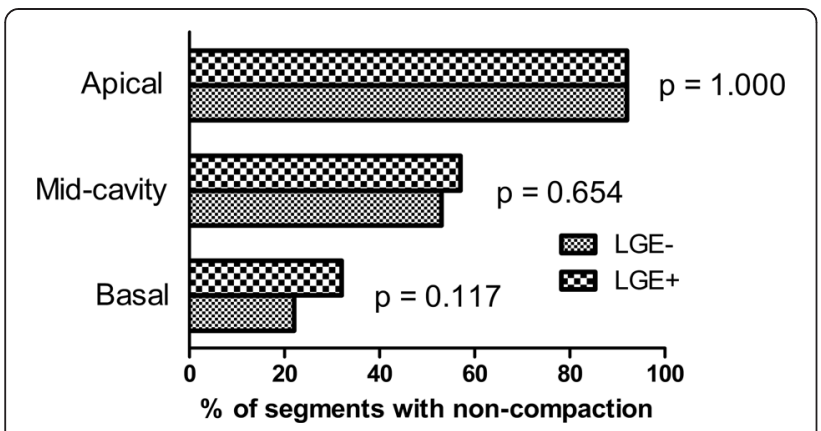

Fig. 2 Anatomical distribution of non-compaction. Comparison of distribution of non-compaction according to region between LGE+ and LGE- children

total cohort. There was no statistically significant difference in the length of follow-up between LGE+ and LGEchildren $(2.6 \pm 2.3$ versus $3.2 \pm 2.3$ years; $p=0.494)$. In LGE+ cohort, adverse events occurred in 6 children, including orthotropic heart transplantation in 2 children and death in 4 children. In LGE- cohort, adverse event was identified in 2 children. Kaplan-Meier analysis showed a significant difference in outcome between LGE+ and LGE- cohorts for cardiac death and heart transplantation (Fig. 4).

\section{Discussions}

In the relatively large cohort study of children with isolated LVNC diagnosed by CMR, we investigated and compared
CMR features and clinical outcomes between LGE+ and LGE- children. The overall occurrence of LGE (25 \%) was lower to that found in previous adult studies $[14,15]$. The LGE + cohort exhibited a more maladaptive LV remodeling and higher incidence of cardiovascular death and heart transplantation.

CMR is showing increasing diagnostic potential in LVNC owing to a comprehensive identification and quantification of the extent of non-compacted myocardium [10, 20, 23]. The utility of CMR with LGE for detecting myocardial fibrosis is well established, which has also been confirmed in our previous report of the adult patient with LVNC [24]. Several studies have described a high prevalence of LGE in patients with LVNC, and LGE has been shown to be associated with ventricular arrhythmias as well as ventricular dysfunction [12-14]. Our previous study have shown that LGE was present in 19 (40\%) of the 47 patients and was more common in patients with ventricular arrhythmias by 24-h Holter electrocardiography recordings [12]. Dodd et al. [25] showed that the amount of trabecular LGE was correlated significantly with LVEF and an independent predictor of LVEF. Nucifora et al. [14] reported LGE was found in $55 \%$ of patients and correlated with clinical severity and ventricular dysfunction. In keeping with their reports, our study showed LGE+ children had more severe LV dysfunction and NYHA functional class.

Similar to previous studies $[12,13]$, the distribution of LGE was strikingly heterogeneous and involved not only non-compacted segments but also normal segments in

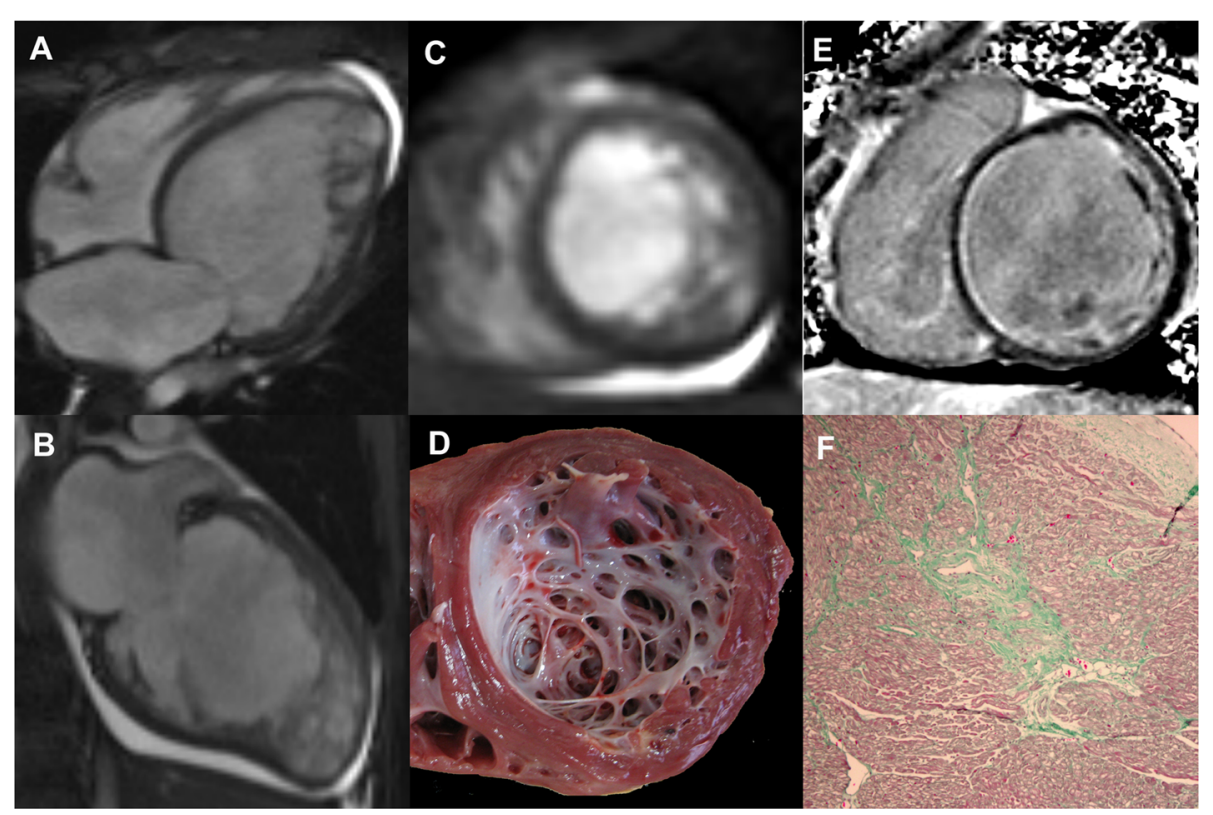

Fig. 3 Representative images in a 14-year-old boy with LVNC underwent heart transplantation. The horizontal (a) and vertical (b) long-axis and short-axis (c) end-diastolic cine images showed prominent trabeculations in anterior, inferior, and lateral segments at apical level. The apical short-axis section of explanted heart $(\mathbf{d})$ revealed prominent trabeculations. Contrast-enhanced image (e) demonstrated subendocardial LGE in the basal septum. Masson's Trichrome staining (f) confirmed the present of replacement fibrosis in corresponding subendocardial area of basal septum (green area); magnification $\times 50$ 


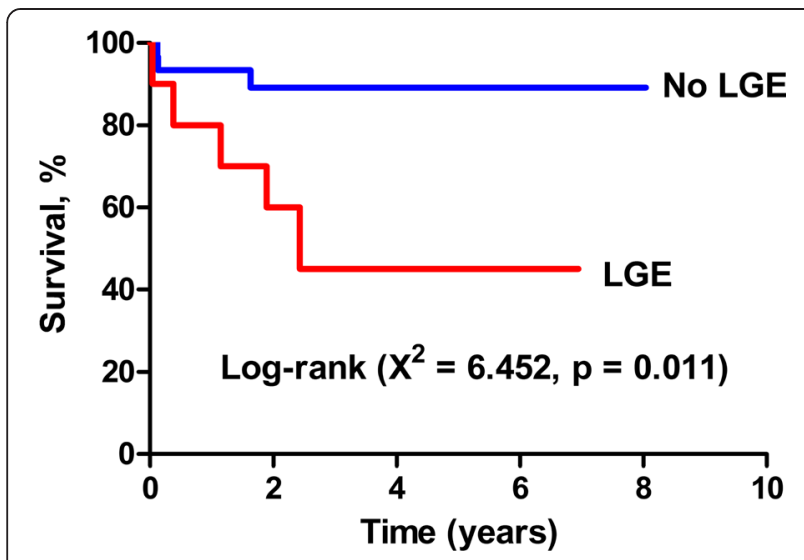

Fig. 4 Adverse events and LGE in children with LVNC. Kaplan-Meier curve shows that the outcomes were worse in children with LGE

the present study. The exact cause of LGE in LVNC remains incompletely determined but is possibly associated with diminished coronary flow reserve, impaired microcirculatory function, coronary artery embolism, and maladaptive LV remodeling. Junga et al. [26] demonstrated restricted myocardial perfusion and decreased flow reserve in areas of ventricular non-compaction in children by positron emission tomography. They concluded the myocardial perfusion defects in non-compacted areas may be the cause of myocardial damage. Jenni et al. [27] found a decreased of coronary flow reserve is not confined to non-compacted segments, but extends to most segments with wall motion abnormalities, thus coronary microcirculatory dysfunction could be associated with ischemia and fibrosis in LVNC. Ridocci-Soriano et al. [28] and our previous studies [12] have demonstrated that coronary artery thrombo-embolism may possibly account for subendocardial or transmural LGE in patients with LVNC. In addition, the adverse cardiac remodeling caused by increased wall stress can result in focal cardiomyocyte necrosis due to augmentation of metabolic demands as well as coronary microcirculatory dysfunction. In the present study, the LV adverse remodeling was more severe in LGE+ children, as evidenced by higher LV volume and sphericity indices. Our study provided further evidence for the potential relationship between myocardial fibrosis and adverse remodeling.

Previous reports on pediatric LVNC associated with congenital heart lesions have suggested that mortality rates were from $15 \%$ to $20 \%$ during follow-up [6, 7]. Recent study of 242 children with LVNC by Brescia et al. [29] showed that 31 children $(12.8 \%)$ died and $13(5.4 \%)$ underwent heart transplantation, resulting in an $18.2 \%$ incidence of death and transplantation in the median 4.0 years of follow-up. Similar to their studies, incidence of death and transplantation was $20 \%$ in mean 3 years of follow-up in our children study cohort. Although the presence of LGE has been shown to be a strong discriminator of adverse outcomes in dilated and hypertrophic cardiomyopathies [30,31], a very limited amount of data has been available on the prognostic value of LGE in children with LVNC. Our study showed a significantly higher rate of cardiac death and heart transplantation in LGE+ children. We propose that the presence of LGE could therefore potentially play an important role in stratification of treatment in children with LVNC. However, this finding should be interpreted with caution owing to the small sample size and lower occurrence of end-point events in the cohort. The pathophysiology and meaning of LGE in children with LVNC need to be elucidated in the future studies.

\section{Limitations}

The present study has several limitations. First, the number of the cohort was still small owing to the relatively rare pediatric entity and a single-center nature. Second, there were potential selection and referral biases in our cohort, given most children clinically referred for CMR investigation had dramatic symptoms that may not be universally applicable to all populations. Third, LGE+ children had more adverse LV remodeling at baseline, which leads to indefinite relationship between the presence of LGE and adverse events. In order to conclusively ascertain incremental prognostic value of LGE in children with LVNC, larger studies with longer follow-up are required.

\section{Conclusions}

In children with LVNC, the LGE is present in up to onefourth of children with LVNC, and LGE+ children exhibited a more maladaptive LV remodeling and higher incidence of cardiovascular death and heart transplantation. The potential clinical utility of LGE in children with LVNC needs to be investigated by larger sample size studies with longer follow-up.

\section{Abbreviations \\ LVNC: Left ventricular non-compaction; CMR: Cardiovascular magnetic resonance; LV: Left ventricular; LGE: Late gadolinium enhancement; NC/C: Non-compacted-to-compacted; EDV: End-diastolic volume; ESV: End-systolic volume; LVEF: Left ventricular ejection fraction; NYHA: New York Heart Association; VT: Ventricular tachycardia; VF: Ventricular fibrillation; ACEl: Angiotensin-converting enzyme inhibitor; ARB: Angiotensin receptor blocker.}

\section{Competing interests}

The authors declare that they have no competing interests.

\section{Authors' contribution}

JP and SZ were responsible for the conception and the design of the study. $\mathrm{HC}$ drafted the manuscript. XC, WX, CC and GY interpreted and analysed the data. $\mathrm{CH}, \mathrm{XC}$ and JW were involved in clinical acquisition of data. ML and HC performed the consensus read of CMR results. JC and SZ helped draft the manuscript. LL helped to acquire and interpret pathologic image. SKP gave critical revision of the manuscript. All authors have seen and approved the final version of the manuscript. 


\section{Author's information}

Pu and Zhao are joint corresponding authors.

\section{Acknowledgments}

We thank the participating cardiologists who contributed patients to the study and who assisted with the clinical follow-up. This study was supported by the Key Projects of National Natural Science Foundation of China (No. 81130029), the National Basic Research Program of China (973 program projects, No. 2013CB531105), and NIHR funding. Dr Prasad is supported by the NIHR Cardiovascular Biomedical Research Unit at the Royal Brompton and Harefield NHS Foundation Trust and Imperial College London, as well as The Rosetrees Trust.

\section{Author details}

${ }^{1}$ Center for Arrhythmia Diagnosis and Treatment, State Key Laboratory of Cardiovascular Disease, Fuwai Hospital, National Center for Cardiovascular Diseases, Chinese Academy of Medical Sciences and Peking Union Medical College, 100037 Beijing, People's Republic of China. ²Department of Radiology, State Key Laboratory of Cardiovascular Disease, Fuwai Hospital, National Center for Cardiovascular Diseases, Chinese Academy of Medical Sciences and Peking Union Medical College, 100037 Beijing, People's Republic of China. ${ }^{3}$ Department of Pathology, State Key Laboratory of Cardiovascular Disease, Fuwai Hospital, National Center for Cardiovascular Diseases, Chinese Academy of Medical Sciences and Peking Union Medical College, 100037 Beijing, People's Republic of China. ${ }^{4}$ Cardiovascular Magnetic Resonance Unit, NIHR Biomedical Research Unit, Royal Brompton Hospital, Sydney Street, SW3 6NP London, UK.

\section{Received: 25 November 2014 Accepted: 14 May 2015}

\section{Published online: 30 May 2015}

\section{References}

1. Oechslin EN, Attenhofer Jost CH, Rojas JR, Kaufmann PA, Jenni R. Long-term follow-up of 34 adults with isolated left ventricular noncompaction: a distinct cardiomyopathy with poor prognosis. J Am Coll Cardiol. 2000;36:493-500.

2. Chin TK, Perloff JK, Williams RG, Jue K, Mohrmann R. Isolated noncompaction of left ventricular myocardium. A study of eight cases. Circulation. 1990;82:507-13.

3. Bagur $\mathrm{RH}$, Lederlin M, Montaudon M, Latrabe V, Corneloup O, Iriart X, et al. Images in cardiovascular medicine. Ebstein anomaly associated with left ventricular noncompaction. Circulation. 2008;118:e662-4.

4. Ichida F, Tsubata S, Bowles KR, Haneda N, Uese K, Miyawaki T, et al. Novel gene mutations in patients with left ventricular noncompaction or Barth syndrome. Circulation. 2001;103:1256-63.

5. Ichida F, Hamamichi Y, Miyawaki T, Ono Y, Kamiya T, Akagi T, et al. Clinical features of isolated noncompaction of the ventricular myocardium: long-term clinical course, hemodynamic properties, and genetic background. J Am Coll Cardiol. 1999:34:233-40

6. Pignatelli RH, McMahon CJ, Dreyer WJ, Denfield SW, Price J, Belmont JW, et al. Clinical characterization of left ventricular noncompaction in children: a relatively common form of cardiomyopathy. Circulation. 2003;108:2672-8.

7. Punn R, Silverman NH. Cardiac segmental analysis in left ventricular noncompaction: experience in a pediatric population. J Am Soc Echocardiogr. 2010;23:46-53.

8. Borreguero LJ, Corti R, de Soria RF, Osende JI, Fuster V, Badimon JJ. Images in cardiovascular medicine. Diagnosis of isolated noncompaction of the myocardium by magnetic resonance imaging. Circulation. 2002;105:E177-8.

9. Petersen SE, Selvanayagam JB, Wiesmann F, Robson MD, Francis JM, Anderson $\mathrm{RH}$, et al. Left ventricular non-compaction: insights from cardiovascular magnetic resonance imaging. J Am Coll Cardiol. 2005;46:101-5.

10. Jacquier A, Thuny F, Jop B, Giorgi R, Cohen F, Gaubert JY, et al. Measurement of trabeculated left ventricular mass using cardiac magnetic resonance imaging in the diagnosis of left ventricular non-compaction. Eur Heart J. 2010;31:1098-104

11. Jassal DS, Nomura CH, Neilan TG, Holmvang G, Fatima U, Januzzi J, et al. Delayed enhancement cardiac MR imaging in noncompaction of left ventricular myocardium. J Cardiovasc Magn Reson. 2006:8:489-91.

12. Wan J, Zhao S, Cheng H, Lu M, Jiang S, Yin G, et al. Varied distributions of late gadolinium enhancement found among patients meeting cardiovascular magnetic resonance criteria for isolated left ventricular non-compaction. J Cardiovasc Magn Reson. 2013;15:20.
13. Dursun M, Agayev A, Nisli K, Ertugrul T, Onur I, Oflaz H, et al. MR imaging features of ventricular noncompaction: emphasis on distribution and pattern of fibrosis. Eur J Radiol. 2010;74:147-51.

14. Nucifora G, Aquaro GD, Pingitore A, Masci PG, Lombardi M. Myocardial fibrosis in isolated left ventricular non-compaction and its relation to disease severity. Eur J Heart Fail. 2011;13:170-6.

15. Ashrith G, Gupta D, Hanmer J, Weiss RM. Cardiovascular magnetic resonance characterization of left ventricular non-compaction provides independent prognostic information in patients with incident heart failure or suspected cardiomyopathy. J Cardiovasc Magn Reson. 2014;16:64.

16. Iwashima S, Ishikawa T, Ohzeki T. Delayed enhancement cardiac MRI in isolated noncompaction of the left ventricular myocardium in a child. Circ J. 2008;72:676-8.

17. Junqueira FP, Fernandes FD, Coutinho AC, De Pontes PV, Domingues RC. Case report. Isolated left ventricular myocardium non-compaction: MR imaging findings from three cases. Br J Radiol. 2009;82:e37-41.

18. Uribe S, Cadavid L, Hussain T, Parra R, Urcelay G, Heusser F, et al. Cardiovascular magnetic resonance findings in a pediatric population with isolated left ventricular non-compaction. J Cardiovasc Magn Reson. 2012;14:9.

19. Muser D, Nucifora G, Gianfagna E, Pavoni D, Rebellato L, Facchin D, et al. Clinical spectrum of isolated left ventricular noncompaction: thromboembolic events, malignant left ventricular arrhythmias, and refractory heart failure. J Am Coll Cardiol. 2014;63, e39.

20. Stacey RB, Andersen MM, St Clair M, Hundley WG, Thohan V. Comparison of systolic and diastolic criteria for isolated LV noncompaction in CMR. JACC Cardiovasc Imaging. 2013;6:931-40.

21. Carluccio E, Biagioli P, Alunni G, Murrone A, Giombolini C, Ragni T, et al. Patients with hibernating myocardium show altered left ventricular volumes and shape, which revert after revascularization: evidence that dyssynergy might directly induce cardiac remodeling. J Am Coll Cardiol. 2006:47:969-77.

22. Cerqueira MD, Weissman NJ, Dilsizian V, Jacobs AK, Kaul S, Laskey WK, et al. Standardized myocardial segmentation and nomenclature for tomographic imaging of the heart. A statement for healthcare professionals from the Cardiac Imaging Committee of the Council on Clinical Cardiology of the American Heart Association. Circulation. 2002;105:539-42.

23. Captur G, Muthurangu V, Cook C, Flett AS, Wilson R, Barison A, et al. Quantification of left ventricular trabeculae using fractal analysis. J Cardiovasc Magn Reson. 2013;15:36.

24. Chaowu Y, Li L, Shihua Z. Histopathological features of delayed enhancement cardiovascular magnetic resonance in isolated left ventricular noncompaction J Am Coll Cardiol. 2011;58:311-2.

25. Dodd JD, Holmvang G, Hoffmann U, Ferencik M, Abbara S, Brady TJ, et al. Quantification of left ventricular noncompaction and trabecular delayed hyperenhancement with cardiac MRI: correlation with clinical severity. AJR Am J Roentgenol. 2007;189:974-80.

26. Junga G, Kneifel S, Von Smekal A, Steinert H, Bauersfeld U. Myocardial ischaemia in children with isolated ventricular non-compaction. Eur Heart J. 1999;20:910-6.

27. Jenni R, Wyss CA, Oechslin EN, Kaufmann PA. Isolated ventricular noncompaction is associated with coronary microcirculatory dysfunction. J Am Coll Cardiol. 2002;39:450-4

28. Ridocci-Soriano F, Estornell-Erill J, Restrepo-Calle JJ, Paya-Serrano R. Isolated non-compaction of the myocardium as a cause of coronary and cerebral embolic events in the same patient. Eur Heart J. 2010;31:727.

29. Brescia ST, Rossano JW, Pignatelli R, Jefferies JL, Price JF, Decker JA, et al. Mortality and sudden death in pediatric left ventricular noncompaction in a tertiary referral center. Circulation. 2013;127:2202-8.

30. Gulati A, Jabbour A, Ismail TF, Guha K, Khwaja J, Raza S, et al. Association of fibrosis with mortality and sudden cardiac death in patients with nonischemic dilated cardiomyopathy. Jama. 2013;309:896-908.

31. Chaowu Y, Shihua Z, Jian L, Li L, Wei F. Cardiovascular magnetic resonance characteristics in children with hypertrophic cardiomyopathy. Circ Heart Fail. 2013;6:1013-20. 